\title{
Erratum: "Analytical solution of the almost-perfect-lens problem" [Appl. Phys. Lett. 84, 1290 (2004)]
}

R. Merlin

FOCUS Center and Department of Physics, The University of Michigan Ann Arbor, MI 48109-1120

(Received 10 November 2003; accepted 7 January 2004)

[DOI: $10.1063 / 1.1789572]$

There is a typographical error in Eq. (4). Also, the results in the electrostatic limit are only applicable to $\mu=1$. More generally, $\lambda$ should be replaced by $\lambda \sqrt{2 /(\mu+1)}$ in the abscissa of the graph and the caption of Fig. 2 as well as in the last part of the paragraph of p. 1292, before the acknowledgments. The correct expression of Eq. (4) is:

$$
H_{y}^{\mathrm{NF}}=\int_{|q|>\omega / c} \mathcal{H}(q) e^{i q x-\sqrt{q^{2}-\omega^{2} / c^{2}}|z+\ell|} d q
$$

For arbitrary $\mu>-1$, the paragraph should read: “ . . provided we make the substitution $2 \pi(d / \lambda) \sqrt{(1+\mu) / 2}=\sqrt{|\sigma|} \ln (2 /|\sigma|)$. Using this expression and Eq. (10) we can easily calculate the lens' resolution. The dependence of $L_{R} / d$ on $(\lambda / d) \sqrt{2 /(\mu+1)}$ is shown in Fig. 2. Explicitly, $4(\lambda / d)^{2} /(\mu+1)=e^{2 \pi d / L_{R}}\left(L_{R} / d\right)^{2}$." 